Original Article

\title{
Comparison of Outcome of Chronic Subdural Hematoma (SDH) In Terms of Recurrence with Drain and without Drain
}

\author{
Ehtisham Ahmed Khan Afridi ${ }^{1}$, Adil Ihsan ${ }^{1}$, Shabana Naz ${ }^{2}$, Gohar Ali ${ }^{3}$, Asghar Ali ${ }^{1}$, Shah Khalid ${ }^{1}$, \\ Abdul Hameed Khan', Mehboob Khan' \\ ${ }^{1}$ Departments of Neurosurgery, Ayub Teaching Hospital, Abbottabad \\ ${ }^{2}$ Department of Pathology, Ayub Teaching Hospital, Abbottabad \\ ${ }^{3}$ Department of Neurosurgery, Bacha Khan Medical College, Mardan - Pakistan
}

\section{ABSTRACT}

Background/Objectives: Chronic subdural hematoma commonly reported in neurosurgical practices. Variations are reported in neurosurgical practices regarding the treatment of chronic subdural hematoma (SDH). This study determined the outcomes of SDH with Drain and without Drain in terms of recurrence and effectiveness.

Material and Methods: A randomized control trial was conducted in the Department of Neurosurgery Ayub Teaching Hospital Abbottabad. Group-A patients were subjected to drainage of chronic subdural hematoma without drain and Group-B patients were subjected to drainage with drain. All patients were followed up to one month for recurrence of subdural hematoma like on CT-scan.

Results: Overall, the recurrence of chronic subdural hematoma in both groups was seen in $16.7 \%$ of patients and the procedures were effective in $83.3 \%$ of patients. In Group A, $79.6 \%$ of patients were successfully treated through burr hole with irrigation, while $20.4 \%$ of patients had recurrent chronic SDH. In Group B, only $13 \%$ of patients had a recurrence, while $87 \%$ of patients were successfully treated with burr with the closed continuous drainage system. An insignificant difference ( $p$-value: 0.302 ) existed between groups for both types of procedures.

Conclusion: The patients who were treated with burr hole and irrigation of the subdural space with normal saline had high recurrence incidence than those with burr hole with closed continuous drainage system.

Keywords: Chronic Subdural Hematoma (CSDH), Burr Hole with Irrigation, Burr Hole with Closed Drainage System, Recurrence.

Corresponding Author: Ehtisham Ahmed Khan Afridi

Department of Neurosurgery, ATH, Abbottabad

E-mail: ehtisham81@gmail.com

Date of Submission: 28-07-2020

Date of Revision: 22-04-2021
Date of Online Publishing: 13-06-2021

Date of Print: 30-06-2021

DOI: 10.36552/pjns.v25i2.459 


\section{INTRODUCTION}

Chronic subdural hematoma is a collection of liquefied hematoma surrounding by capsule within the subdural space, ${ }^{1}$ usually resulting from rupture of cortical veins, traumatic in nature and most likely appear in patients with cerebral atrophy, history of alcoholism or patients taking anticoagulants, increased capillary fragility, abnormal gait, and patients with increase motor deficits. Other possible causes in the formation of chronic subdural hematoma are arteriovenous malformations, cerebral aneurysms, hemorrhagic diathesis, brain tumors, and infectious diseases. ${ }^{2,3}$ Incidence of chronic subdural hematoma is 7.4 out of 1000000 population, ${ }^{2}$ while another study showed it to be 5 cases per 100000 in the general population. ${ }^{4}$

In patients above 50 years of age, the brain parenchyma becomes atrophic with reductions as low as 200 grams and consequently increased dural space. Hemorrhage can easily occupy this space surrounding the brain without raising ICP (intracranial pressure). The hematoma forms an outer capsular layer. It absorbs subdural fluid, and also develops vascular channels, that easily bleed. Because of loose cellular junctions. There is a balance between the expansion of hematoma and its absorption, and as this balance disrupts, the expanding hematoma produces symptoms. Patients with chronic subdural hematoma usually present with complaints of headache, weakness in one half of the body, unstable gait, balance loss, deteriorating memory, recurrent falls, confusion, lethargy, and dizziness. ${ }^{2}$ Chronic Subdural Hematoma commonly reported in neurosurgical practices. Variations are reported in neurosurgical practices regarding the treatment of chronic subdural hematoma (SDH). This study aimed to evaluate the outcomes of SDH with drain and without drain in terms of recurrence and effectiveness.

\section{MATERIAL AND METHODS}

\section{Study Design and Setting}

A randomized control trial was conducted at the Department of Neurosurgery Ayub Teaching Hospital Abbottabad from October 2012 to March 2013. Formal approval from the ethical committee was obtained to conduct the study.

\section{Sample Size and Technique}

A total of 54 patients were included in each group using $9.3 \%$ the proportion of recurrence of subdural hematoma using drain and $24 \%$ proportion of recurrence after not using any drain. ${ }^{17}$ A 95\% confidence interval and $90 \%$ power of the test were considered for the WHO sample size calculations. Consecutive non-probability sampling was considered.

\section{Inclusion Criteria}

All patients above the age of 20 years from both sexes with chronic subdural hematoma on CT scans, were included in the study.

\section{Exclusion Criteria}

Patients with any history of procedure for cerebrospinal fluid diversion, bleeding disorders, recurrence of subdural hematoma, chronic diseases of liver or kidneys or were on cardiac medication or anticoagulants were not included in the study to avoid bias.

\section{Patient Groups}

Patients included admitted through OPD and emergency. They were admitted to the neurosurgery department where written and informed consents were taken. History and physical examination of each patient followed by neurological assessment were also carried out.

Two groups were made where patients were randomly added by the lottery method. 
Group A patients were subjected to drainage of chronic subdural hematoma without drain.

Group B patients were subjected to drainage with drain.

All patients were followed up to one month for recurrence of subdural hematoma on CT-Scan. All the surgeries were performed by a single experienced neurosurgeon.

\section{Data Collection and Analysis}

Data collected for patients' age, sex, type of procedure and recurrence, through a predesigned proforma. All data entered, processed, and analyzed with SPSS (version 24). Descriptive statistics were used to calculate the standard deviation. Frequency and percentages were presented for gender and effectiveness. Chisquare test was used for comparison in both groups with a $p$-value of $\leq 0.05$ as significant.

\section{RESULTS}

A total of 108 patients were studied, who met the inclusion criteria like patients having age above 20 years, patients of either gender and symptomatic patients with a diagnosis of chronic subdural hematoma on CT-Scan or MRI Brain.

\section{Gender Distribution}

Among these patients 104 (96.3\%) were male and $4(3.7 \%)$ were female patients.

\section{Age Range}

Age range was 23 years to 85 years with a mean age of $64.04 \pm 10.29$ years.

\section{Recurrence of Chronic Subdural Hematoma}

Patients were randomly distributed into two groups, and each group includes 54 patients. As a whole, the recurrence of chronic subdural hematoma in both groups was seen in 18 (16.7\%) patients and the procedures were effective in 90 (83.3\%) patients and were not effective in $16.7 \%$ of patients. Tables 1 and 2 mention the detail.

In Group A, 43 (79.6\%) of patients were successfully treated through burr hole with irrigation, while 11 (20.4\%) of patients had recurrent chronic SDH, so the procedure was effective in $79.6 \%$ of patients and was not effective in $20.4 \%$ of patients.

Table 1: Comparison of both groups in terms of outcome and recurrence.

\begin{tabular}{|c|c|c|c|c|}
\hline & \multicolumn{2}{|c|}{$\begin{array}{c}\text { Recurrence of } \\
\text { Subdural Hematoma }\end{array}$} & \multirow[t]{2}{*}{ Total } \\
\hline & & Yes & No & \\
\hline \multirow{6}{*}{ Groups } & \multirow{2}{*}{$\begin{array}{l}\text { Burr Hole with } \\
\text { Irrigation }\end{array}$} & 11 & 43 & 54 \\
\hline & & $20.4 \%$ & $79.6 \%$ & $100.0 \%$ \\
\hline & $\begin{array}{l}\text { Burr Hole with } \\
\text { Closed }\end{array}$ & 7 & 47 & 54 \\
\hline & $\begin{array}{l}\text { Drainage } \\
\text { System }\end{array}$ & $13.0 \%$ & $87.0 \%$ & $100.0 \%$ \\
\hline & \multirow{2}{*}{ Total } & 18 & 90 & 108 \\
\hline & & $16.7 \%$ & $83.3 \%$ & $100.0 \%$ \\
\hline \multicolumn{2}{|c|}{ Chi-Square \&p-value } & \multicolumn{3}{|c|}{$\begin{array}{l}\chi^{2}=1.0677 ; p \text { value }=0.302 \\
\text { (insignificant result) }\end{array}$} \\
\hline
\end{tabular}

Table 2: Effectiveness of both Procedures.

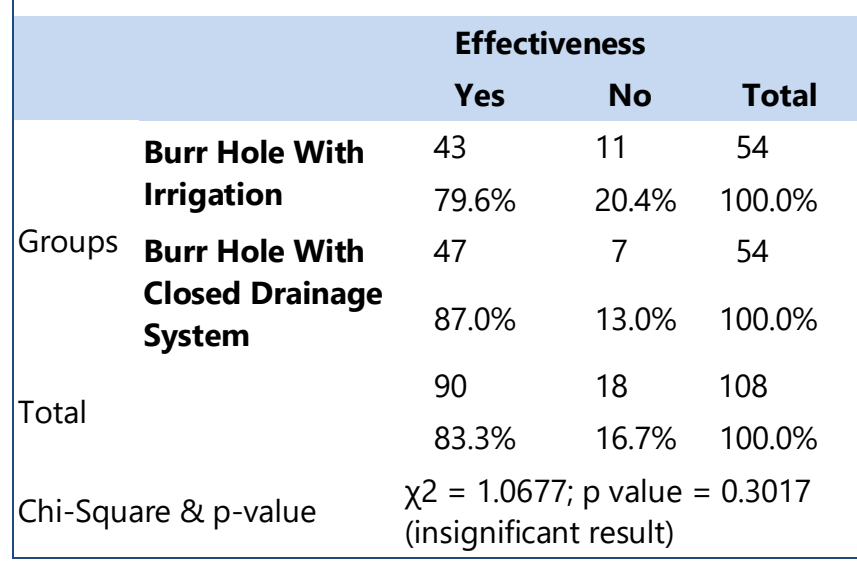

In Group B, only 7 (13.0\%) of patients had recurrence, while 47 (87.0\%) of patients were 
successfully treated with burr with a closed continuous drainage system, so the procedure was effective in $87.0 \%$ of patients and was not effective in only $13.0 \%$ of patients. An insignificant difference ( $p$-value: $0.302>0.05$ ) existed between groups for both types of procedures, that is, burr hole with irrigation and burr hole with the closed drainage system.

\section{DISCUSSION}

Variations are reported in neurosurgical practices regarding the treatment of Chronic Subdural Hematoma (SDH). This study determined the outcomes of SDH with Drain and without Drain in terms of recurrence and effectiveness. We observed that the burr hole with closed drainage is better in terms of effectiveness. Minor differences existed in the treatment plans in different institutions. This underscores a fact that much is still not understood about this medical condition. There is substantial documentation about treatment differences for chronic SDH, around the world, as shown in their data regarding the use of drains, mobilization of patients, and positioning of the head during and after surgery. ${ }^{6,7}$

Our study showed that $93.3 \%$ of patients presented with chronic subdural hematoma were male, and only $3.7 \%$ of patients were female with a mean age of presentation was $64.04 \pm 10.298$ years, which closely matches the results shown by Sousa et $\mathrm{al}_{1}{ }^{8}$ in which they reported that $87.5 \%$ of patients were male and only $12.5 \%$ of patients were female with a mean age of presentation was $64.3 \pm 15.9$ years. In our study, males were involved in chronic subdural hematoma because of our society, where males are more exposed to traffic and outdoor activities as compared to females. The elderly patients were involved because of extensive brain atrophy with age, or certain circumstances resulting in decrease intracranial pressure like ventriculoperitoneal shunting are vulnerable to develop a chronic subdural hematoma. Stretch on the fragile bridging veins in the atrophied brain gives way to hematoma formation. Later followed by deposition of fibrin, organization of clot, fibrinolysis through enzymes, and then liquification of the clot. ${ }^{9.10}$ In elderly patients, with the atrophied brain, there is an $11 \%$ increase in the extracerebral volume. The brain has more space for movement inside the skull and thus more chances of injuries to the bridging vessels. Males have more exposure to injuries, hence the higher percentage of males in both the studies.

Recurrence of CSDH in our study was $16.7 \%$, which closely matches the results of the study done by Khan et $\mathrm{al}^{12}$ who showed in his study it to be $16.6 \%$. Recurrence is high because of cerebral re-expansion after drainage. Reexpansion is greater in patients below 70 years than those above 70 years of age. Re-expansion of the brain after surgery was also affected by presence of medical conditions like brain infarcts or air in the subdural space. Erol et $\mathrm{al}^{13}$ suggested that recurrence rate varied with the amount of removal of the semisolid component of SDH and by the dilution of endogenous fibrinolytic agents. Similarly, Oh et $\mathrm{al}^{14}$ and Mulligan et $\mathrm{al}^{15}$ showed in their studies, the recurrence of CSDH to be $16 \%$ and $20 \%$ respectively, which closely matches the results of our study. Our study showed that, the patients in Group A, who were operated through Burr Hole with Irrigation, the procedure was effective in $79.6 \%$ of patients and recurrence was noted in $20.4 \%$ of patients, while in Group B, who were operated through Burr with Closed Drainage system, the procedure was effective in $83.3 \%$ of patients, and recurrence was noted in only $16.7 \%$ of patients, which closely matches the results of a study done by Santarius et $\mathrm{al}^{16}{ }^{16}$ who showed that the recurrence of chronic subdural hematoma was $24 \%$, who were operated through Burr Hole with Irrigation without Drain and 9.3\% recurrence was reported in patients who were operated through Burr Hole with Drain. The fact was explained by Gurunathan et $\mathrm{al}_{1}{ }^{17}$ they mentioned 
that the reduction of residual air volume in the subdural space by keeping drain in the subdural cavity is a suitable mean to avoid recurrence of chronic subdural hematoma. Similarly, Ahmed et $\mathrm{al}^{4}$ showed that the recurrence of chronic subdural hematoma is common in patients who were operated through burr hole without drain i.e., $23 \%$, in comparison to those patients who were operated with closed-system drainage i.e., $16 \%$.

Although we did not find any statistical difference in the complication rate in groups, the use of drains is still a matter of surgical concern for neurosurgeons around the world. Our study brought similar results to the other series regarding the surgical techniques, baseline characteristics, general management, distribution, and frequency of complications. Our study results have supported the use of drains in the surgical treatment of chronic SDH.

\section{CONCLUSION}

The surgical outcome was fairly good for continuous drainage after burr hole of chronic subdural hematoma. Closed continuous drainage showed lower recurrence rates than the patients who were treated with burr hole and saline lavage. We concluded that chronic subdural hematoma can be efficiently evacuated by closed continuous drainage after burr hole. It is a safer simpler and more efficient way of treatment of chronic subdural hematomas, that takes a shorter time and has lower chances of recurrence.

\section{REFERENCES}

1. Lee KS, Shim JJ, Yoon SM, Doh JW, Yun IG, Bae HG. Acute-on-chronic subdural hematoma: Not Uncommon Events. J Korean Neurosurg Soc. 2011; 50 (6): 512-6.

2. Mostofi K, Marnet D. Percutaneous Evacuation for treatment of Subdural Hematoma and outcome in 28 patients. Turkish Neurosurg. 2011; 21 (4): 522-6.

3. Soto-Granados M. Treatment of chronic subdural hematoma through a burr hole. Cirugia y cirujanos, 2010; 78 (3): 203-7.

4. Ahmed S, Agrawal D, Kale SS, Mahapatra AK. A Comparative study of treatment of chronic subdural hematoma - burr hole drainage versus continuous closed drainage. Indian Journal of Neurotrauma, 2011; 8 (1): 17-24.

5. Lee KS. Natural history of chronic subdural haematoma. Brain Inj. 2004; 18 (4): 351-8.

6. Han HJ, Park CW, Kim EY, Yoo CJ, Kim YB, Kim WK. One vs. Two Burr hole craniostomy in surgical treatment of chronic subdural hematoma. J Korean Neurosurg Soc. 2009; 46: 87-92.

7. Torihashi K, Sadamasa N, Yoshida K, Naurumi O, Chim M, Yamagata S. Independent predictors for recurrent chronic subdural hematoma: a review of 343 consecutive surgical cases. Neurosurgery, 2008; 63: 1125-9.

8. Senturk S, Guzel A, Bilici A, Takmaz I, Guzel E, Aluclu MU, et al. CT and MR Imaging of chronic subdural haematoma: A comparative study. Swiss MED WKLY. 2010; 140 (23 - 24): 335-40.

9. Gelabert-Gonzalez M, Iglesias-pais M, Garcia Allut A, Martinez-Rumbo R. Chronic subdural haematoma: Surgical treatment and outcome in 1000 cases. Clin Neural Neurosurg. 2005; 107: 2239.

10. Ducruet AF, Grobelny BT, Zacharia BE, Hickman ZL, Derosa $\mathrm{PL}$, Anderson $\mathrm{K}$ et al. The surgical management of chronic subdural hematoma. Neurosurg Rev. 2012; 35 (2): 155-169.

11. Baechli H, Nordmann A, Bucher HC, Gratzl O. Demographics and prevalent risk factors of chronic subdural haematoma: results of a large singlecenter cohort study. Neurosurg Rev. 2004 Oct; 27 (4): 263-6.

12. Khan RD, Ali M, Ali M, Rizwan U. The etiology, symptomatology and surgical outcome in 60 cases of Chronic Subdural Hematoma. JPGMI. 2004; 2.

13. Erol FS, Topsakal C, Faik Ozveren M, Kalpan $M$, Tiftikel MT. Irrigation VS Closed Drainage in the treatment of Chronic Subdual Hematoma. J Clin Neurosci. 2005; 12: 261-3.

14. Oh HJ, Lee KS, Shim JJ, Yoon SM, Yun G, Bae HG. Postoperative course and recurrence of chronic subdural hematoma. J Korean Neurosurg Soc. 2010; 48 (6): 518-23.

15. Mulligan P, Raore B, Liu S, Olson JF. Neurological 
and functional outcomes of subdural hematoma evacuation in patients over 70 years of age. J Neurosci Rural Pract. 2013; 4: 250-6.

16. Thapa A, Agrawal B. Mount Fuji Sign in Tension Pneumocphalus. Indian J Neurotrauma, 2009; 6:
161-2.

17. Gurunathan J. Treatment of chronic subdural hematoma with burr whole craniostomy and irrigation. Indian J Neurotrauma, 2005; 2 (2): 12730.

\section{Additional Information}

Disclosures: Authors report no conflict of interest.

Ethical Review Board Approval: The study was conformed to the ethical review board requirements.

Human Subjects: Consent was obtained by all patients/participants in this study.

\section{Conflicts of Interest:}

In compliance with the ICMJE uniform disclosure form, all authors declare the following:

Financial Relationships: All authors have declared that they have no financial relationships at present or within the previous three years with any organizations that might have an interest in the submitted work.

Other Relationships: All authors have declared that there are no other relationships or activities that could appear to have influenced the submitted work.

\section{AUTHORS CONTRIBUTIONS}

\begin{tabular}{|l|l|l|}
\hline Sr.\# & Author's Full Name & Intellectual Contribution to Paper in Terms of: \\
\hline 1. & Ehtisham Ahmed Khan Afridi & 1. $\quad$ Study design, manuscript writing and methodology. \\
\hline 2. & Adil Ihsan & 2. Paper writing, referencing, and data calculations. \\
\hline 3. & Shabana Naz, Gohar Ali & 3. $\quad$ Data collection and calculations. \\
\hline 4. & Asghar Ali, Shah Khalid & 4. Analysis of data and interpretation of results etc. \\
\hline 5. & Abdul Hameed Khan & 5. Literature review. \\
\hline 6. & Mehboob Khan & 6. Analysis of data. \\
\hline
\end{tabular}

